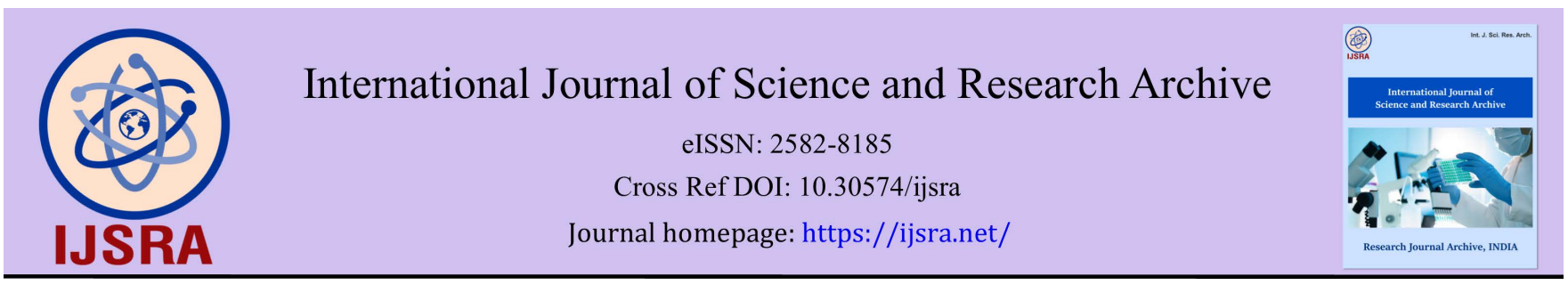

(RESEARCH ARTicle)

\title{
One month safety study of ExoFlo injection for the treatment of lumbar or cervical radiculopathy in the epidural space
}

\author{
Andrew Phillips *, Anita Wong, Grace Chen, Jacob LaSalle and Jonathann Kuo
}

Hudson Medical, 281 Broadway New York, New York 10007.

International Journal of Science and Research Archive, 2021, 04(01), 119-124

Publication history: Received on 13 October 2021; revised on 23 November 2021; accepted on 25 November 2021

Article DOI: https://doi.org/10.30574/ijsra.2021.4.1.0178

\begin{abstract}
This One month safety study addresses any potential risks behind the use of bone marrow-derived mesenchymal stem cell extracellular vesicle isolate product (ExoFlo Direct Biologics) as a treatment for cervical and lumbar radiculopathy. Ten healthy adults were treated with ExoFlo injections for cervical radiculopathy $(n=5)$ and lumbar radiculopathy $(n=5)$. Follow up occurred twenty-four hours, three days, one week, three weeks and one month post injection. By the one month follow-up, the average patient improved 55\% in BPI, 55.2\% in QD, 25.4\% in UEFS, $19.75 \%$ in ODI and $26 \%$ in LEFS. There were no complications or adverse events by the end of the study and no patient exhibited worsening radiculopathy. Patients will continue to be followed for at least six months post injection.
\end{abstract}

Keywords: Exosomes; Regenerative medicine; MSC; Extracellular vesicle isolate product; Radiculopathy; Epidural space

\section{Introduction}

Lumbar and cervical radiculopathies are one of the most widespread neurological complaints that cause significant pain and disability. Radicular disease is prevalent in both men and women, with age and manual labor such as heavy lifting being primary risk factors. Symptoms typically result from inflammation and the irritation of a spinal nerve caused by a compressive force [1]. In younger patients, the most frequent causes for nerve impingement are due to disc trauma and herniation. For older patients in the fifth decade and beyond, disc degeneration and foraminal narrowing from arthritic change become the likely cause of impingement [2]. Patients experiencing lumbar or cervical radiculopathy commonly present with sharp, shooting pain that radiates down to the respective lower or upper extremities. Frequently, radiculopathy is also associated with weakness in the muscles innervated by the affected nerve.

Existing treatments for lumbar and cervical radiculopathy involve initial conservative approaches including physical therapy, chiropractics, acupuncture, manual therapy, NSAIDs and anticonvulsants [3]. Pain injections such as corticosteroid injections have also been utilized in standard care for radiculopathy due to the strong short-term relief received, but patients are often left with limited long-term relief [4]. Duration of relief aside, epidural corticosteroid injections have also been associated with a slew of health risks, including reduction of bone mass density (BMD), increased risk for vertebral fractures, avascular necrosis and heightened blood glucose levels $[5,6,7,8]$. This ultimately demonstrates the inadequacy of corticosteroid injections as a viable and safe intervention option for chronic disorders. Further surgical interventions such as discectomies or spinal fusions may be considered based on the success of conservative treatments and the severity of a patient's pain; however, overall failure rate of lumbar spine surgery was estimated to be $10 \%-46 \%$ [9].

\footnotetext{
* Corresponding author: Andrew Phillips

Hudson Medical, 281 Broadway New York, New York 10007.
}

Copyright $\Subset 2021$ Author(s) retain the copyright of this article. This article is published under the terms of the Creative Commons Attribution License 4.0. 
There is growing interest in the use of PRP and other orthobiologics in epidural injections. It has been documented that platelet lysate injected into the epidural space for the treatment of lumbar radiculopathy results in significant improvement in pain [10]. Nonetheless, given the lack of any standardized processing method, its results could vary highly in safety and efficacy. There is also speculated therapeutic potential of using mesenchymal stem cell-based regenerative treatments based on their hypothesized ability to replenish specific cells and modulate the regenerative environment via anti-inflammatory and immunomodulatory mechanisms. However, issues exist with any autologous cell source including cell quantity and donor viability. Additionally, there are potential safety concerns when injecting into the epidural space including tumor formation, inappropriate stem cell migration, or micro-emboli if trapped in capillary beds $[11,12]$. As opposed to living BM-MSCs, it is understood that the therapeutic efficacy of BM-MSCs stem from their secretion of EVs including exosomes [13].

In recent years, much attention has been drawn to the paracrine mechanism of exosomes and their potential usage in acellular therapies. Exosomes are defined as "extracellular vesicles released on exocytosis of multivesicular bodies (MVBs) filled with intraluminal vesicles (ILVs)" [14]. These 30-150nm-sized bi-phospholipid membrane-enclosed structures contain growth factors, signaling lipids, and nucleic acids (mRNAs and miRNAs) that contribute to their paracrine anti-inflammatory effects in recipient cells [15]. The use of ExoFlo has been studied for several disease indications such as idiopathic pulmonary fibrosis, amyotrophic lateral sclerosis, and joint pathology [13,16,17]. Most recently, exosomes were utilized in patients hospitalized with severe COVID-19 infection and demonstrated profound reversal of hypoxia, immune reconstitution, and downregulation of cytokine storm [18]. MSC-derived exosomes may provide acellular treatment options as an alternative to traditional MSC therapy. The primary objective of this IRB study was to determine the safety of and consider the clinical efficacy of utilizing a single interlaminar epidural injection of BM-MSC-derived exosomes (ExoFlo) to treat lumbar and cervical radiculopathy.

\section{Methods}

\subsection{Study Design}

This study was a prospective, open-label, non-randomized IRB approved safety study of a single ExoFlo injection for the treatment of cervical radiculopathy $(n=5)$ and lumbar radiculopathy $(n=5)$ due to intervertebral disc herniation. Participants were not subjected to any costs for the study product and study services provided. Reimbursement was not offered for involvement in the study. The study protocol was approved by the Institute of Regenerative and Cellular Medicine IRB, Protocol number: HM-EF-001, IRB approval number: IRCM-2020-274. All participants were counseled and presented with informed consent documentation per IRB requirements. Ten (10) healthy adult subjects were enrolled in the study, which was held at a single center (Hudson Medical). All subjects had symptoms of radicular pain for years and MRIs that exhibited mild to moderate herniated nucleus pulposus with neural foraminal compression. In conjunction, medical history reviews and physical examinations were performed. 24 hours prior to the injection, baseline measures were obtained through the Brief Pain Inventory (BPI), Upper Extremity Functional Scale (UEFS), QuickDash (QD), Lower Extremity Functional Scale (LEFS) and ODI (Oswestry Disability Index). These same outcome measures were obtained 24 hours, 3 days, 1 week, 3 weeks and 1 month post-injection through the Qualtrics system. Phone calls and emails were conducted at the aforementioned follow up points to discuss any potential adverse events and/or improvement in symptoms.

\subsection{Therapeutic Description}

ExoFlo contains 10-80 billion extracellular vesicles comprised of proteins, mRNA, miRNA, growth factors and signaling lipids. These secreted extracellular vesicles are derived from mesenchymal stem cells from the bone marrow of a single young and healthy female donor. Donor bone marrow screening was done through a Clinical Laboratory Improvement Amendments (CLIA) licensed laboratory, which also helped to test for any infectious attributes or viruses. Under Current Good Manufacturing Practice (cGMP) regulations, ExoFlo sterilization was attained through $0.2 \mu \mathrm{m}$ ultrafiltration, instead of radiation, to maintain the highest safety profile. The ExoFlo is frozen at $-40^{\circ}$ to $-80^{\circ} \mathrm{C}$ to ensure bioactive stability and then thawed to room temperature prior to the injection.

\subsection{Patient Demographics}

Eligible patients who met our inclusion criteria were men and women at least 18 years of age or older. Patients who had histories of adverse reactions to epidural steroid injections, ongoing malignant disorder, severe stenosis, cauda equina symptoms, myelomalacia or multifactorial diagnoses of radiculopathy were excluded. Those who were currently pregnant were also excluded. The final chosen subjects are described below in Table 1. 
Table 1 Description of Patient Demographics

\begin{tabular}{|l|c|c|}
\hline & Average & Range \\
\hline Age $(\mathrm{n}=10)$ & 44.3 & $35-64$ \\
\hline Areas Treated & \multicolumn{2}{|c|}{ Cervical Spine= 5 Lumbar Spine=5 } \\
\hline
\end{tabular}

\subsection{Injection Technique}

All injections were performed by the principal investigator under standard sterile conditions. Under fluoroscopic guidance, a 22-gauge 3.5 inch epidural Tuohy needle was advanced into the epidural space using the loss of resistance technique. 2-3 mL of Omnipaque 180 contrast was then injected under live fluoroscopic view to confirm placement in the epidural space. A/P and lateral fluoroscopic views were saved. After repeated negative aspiration for blood or CSF, a $3 \mathrm{~mL}$ solution of $2 \mathrm{~mL}$ Direct Biologics ExoFlo and $1 \mathrm{~mL}$ of normal saline was injected. For patients with cervical radiculopathy, an interlaminar epidural injection with approach at the C5-6 or C6-7 level was used. For patients with lumbar radiculopathy, an interlaminar epidural injection with approach the L4-5 or L5-S1 level was used.
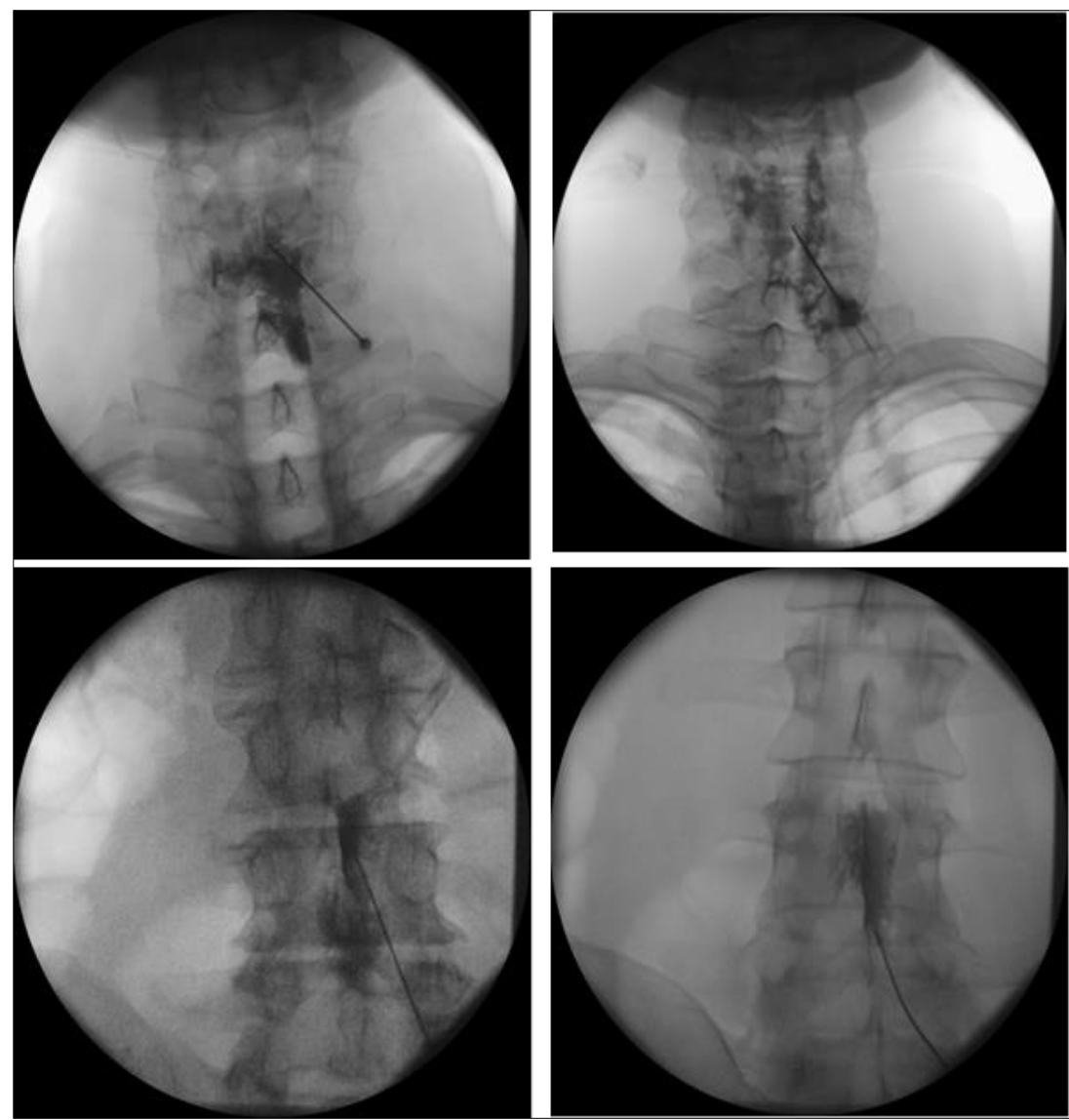

Figure 1 Fluoroscopic images of ExoFlo injections in the cervical (top 2 images) and lumbar (bottom 2 images) epidural space

\section{Results}

Following the injection, every participant was followed up 24 hours, 3 days, 1 week, 3 weeks and 1 month with outcome measures and contacted to review any potential adverse events from the use of ExoFlo. One (1) participant, who presented with lumbar radiculopathy, was released from the study due to inadequate follow up after the 5-day contact point. Another subject was enrolled in their place, per the Institute of Regenerative and Cellular Medicine IRB. At 24 hours post injection, two patients experienced slightly increased pain and four patients noted pain and soreness to the injection site. These adverse events were resolved by the 3-day follow up point. One subject experienced 2 headaches, one at the 3-day follow up point and another 5 days after the injection. The second headache resolved on the same day 
of onset and the patient denied any other headaches throughout the remainder of the study period. There were no other reported adverse side effects, including fever, chills, headaches, dizziness, nausea and vomiting, from the participants following the injection. By the 1-month follow up point, no patient exhibited any worsening cervical or lumbar radiculopathy from the ExoFlo injection.

\section{Discussion}

This preliminary IRB study on the treatment of cervical and lumbar radiculopathy demonstrated that the use of $2 \mathrm{~mL}$ BM-MSC-derived EV product (ExoFlo) can be administered safely through a single interlaminar epidural injection. A few patients experienced post-procedural discomfort including a mild increase in pain and injection-site soreness that were resolved within a few days of the injection. There were no reported complications or major adverse events and no evidence of worsening cervical or lumbar radiculopathy following the ExoFlo injection. At the one month follow-up point, the average patient improved 55\% in BPI , 55.2\% in QD, 25.4\% in UEFS, $19.75 \%$ in ODI and 26\% in LEFS.

There is evidence to suggest that the clinical efficacy of BM-MSCs can be attributed to their release of acellular paracrine factors that modulate inflammatory responses [17]. Current stem cell technologies utilize very small numbers of viable stem cells harvested from either a patient's own bone marrow or adipose tissue, or from allogeneic sources such as placenta, amniotic fluid, and umbilical cord blood. However, there are safety concerns including potential tumor formation, inappropriate stem cell migration, or micro-emboli if trapped in capillary beds [11,12]. The use of BM-MSCderived EVIP holds great promise in providing a significantly higher, more efficacious, and safer dose of exosomal content to recipient cells [18]. In comparison with existing treatments for radiculopathies, the multi-targeted approach of exosome-based therapeutics offers a clear advantage. The immunomodulatory proteins of exosomes immediately take effect within recipient cells to decrease nerve inflammation. These proteins also stimulate production of antiinflammatory chemokines, cytokines, and other signaling molecules that are subsequently released from recipient cells. At the same time, the nucleic acid content of exosomes (mRNAs and miRNAs) are translated or function to inhibit proinflammatory signals. In combination, the multifold downstream effects of exosomes create a reparative environment [17].

This is the first known clinical study to date using BMC-MSC-derived exosomes as treatment for lumbar and cervical radiculopathy. In consideration of the superior safety profile and efficacy of BM-MSC-derived EVIP, it is reasonable to pursue further evaluation of BM-MSC-derived exosome usage in the epidural space for treatment of inflammatory-based spinal pathologies in larger clinical trials.

\section{Conclusion}

Ten healthy adult subjects each received a single injection of BM-MSC EVIP (ExoFlo Direct Biologics) in the interlaminar epidural space for cervical and lumbar radiculopathy. By the 1-month end point, follow up was successful in all subjects for the exception of one patient in the lumbar radiculopathy cohort, who was ultimately replaced. Follow up was done through phone call, email and outcome measures to determine the safety and efficacy of this novel intervention. Adverse events that manifested include 24 hours of increased pain in two patients, 24 hours of soreness and pain to the injection site in four patients, and headaches for one patient. Limitations identified in this study include the short 1-month follow up end point, small study sample, and lack of a control group for randomization. Overall, results in this IRB study demonstrate that the use of exosomes in the interlaminar epidural space was safe and well tolerated. All of the study participants are chronic pain patients established to the practice who have undergone different spinal injection treatments in the past including epidural steroid injections with mild to moderate relief of symptoms in the past. Our average subject experienced promising preliminary pain improvement of 55\% in BPI , 55.2\% in QD, 25.4\% in UEFS, $19.75 \%$ in ODI and 26\% in LEFS with the ExoFlo injection at the 1 month mark. Many of them noted that the pain relief was more significant than with previous traditional epidural steroid injections. A few subjects even exhibited $80 \%$ or more improvement in their pain severity according to BPI assessments by the 1 month follow-up point. Given the lack of significant adverse events, the encouraging data points thus far, and this being the first known study of the usage of extracellular vesicles in the epidural space, we will be pursuing a dedicated efficacy study for further examination of use of BM-MSC EVIP in the treatment of cervical and lumbar radiculopathy.

\section{Compliance with ethical standards}

\section{Disclosure of conflict of interest}

The authors declare they have no competing interests in this study. 


\section{Statement of ethical approval}

The investigators performed research on human participants through ethical procedures with IRB review prior to initiating treatment implementation.

\section{Statement of informed consent}

Informed consent was obtained from all participants prior to trial commencement.

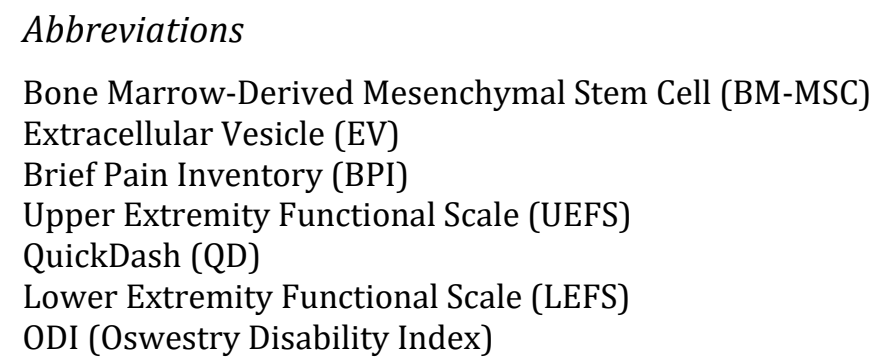

\section{References}

[1] Berry, James A, et al. "A Review of Lumbar Radiculopathy, Diagnosis, and Treatment." Cureus. Oct. 2019; 11(10).

[2] Magnus, Warren et al. Cervical Radiculopathy. StatPearls Publishing, Treasure Island (FL). 2021.

[3] Wong, JJ, et al. Clinical practice guidelines for the noninvasive management of low back pain: a systematic review by the Ontario Protocol for Traffic Injury Management (OPTIMa) Collaboration. Eur J Pain. Feb 2017; 21(2): 201-216.

[4] Salahadin Abdi, et al. Epidural steroids in the management of chronic spinal pain: a systematic review. Pain Physician. Jan. 2007; 10(1): 185-212.

[5] Kim, Minsoo, et al. Effect of Medications and Epidural Steroid Injections on Fractures in Postmenopausal Women with Osteoporosis." Medicine. Jun. 2019; 98(26).

[6] Kerezoudis, Panagiotis, et al. The Effect of Epidural Steroid Injections on Bone Mineral Density and Vertebral Fracture Risk: A Systematic Review and Critical Appraisal of Current Literature. Pain Medicine. Mar. 2018; 19(3): 569-579.

[7] Laroche, Michel, et al. Osteonecrosis of the Femoral and Humeral Heads After Intraarticular Corticosteroid Injections." The Journal of Rheumatology. Apr. 1990; 17(4): 549-51.

[8] Younes, Mohamed, et al. Systemic Effects of Epidural and Intra-Articular Glucocorticoid Injections in Diabetic and Non-Diabetic Patients. Joint Bone Spine. Jul. 2007; 74(5): 472-76.

[9] Thomson S. Failed back surgery syndrome - definition, epidemiology and demographics. Br J Pain. 2013 Feb; 7(1): 56-9.

[10] Centeno, Christopher et al. The use of lumbar epidural injection of platelet lysate for treatment of radicular pain. Journal of experimental orthopaedics. Nov. 2017; 4(1): 38-25.

[11] Bligh, Richard. "Treatment of Idiopathic Pulmonary Fibrosis with an Extracellular Isolate Product." International Journal of Science and Research Archive. May. 2021; 02(02): 231-236.

[12] Choumerianou, Despoina M, et al. Stem cells: promises versus limitations. Tissue Eng Part B Rev. Mar. 2008; 14(1): 53-60.

[13] Li Xiaohong, et al. Mesenchymal stem cells in idiopathic pulmonary fibrosis. Oncotarget. May. 2017; 8(60): 102600-102616.

[14] Emanuele, Cocucci, Meldolesi, Jacopo. Ectosomes and exosomes: shedding the confusion between extracellular vesicles. Trends Cell Biol. Jun. 2015; 25(6): 364-72.

[15] Lee, Changjin, et al. Exosomes mediate the cytoprotective action of mesenchymal stromal cells on hypoxiainduced pulmonary hypertension. Circulation. Nov. 2012; 126(22): 2601-11. 
[16] Crose, Joshua James. Treating amyotrophic lateral sclerosis with a bone marrow derived mesenchymal stem cell extracellular vesicles - A case report. International Journal of Science and Research Archive. May. 2021; 02(02): 167-171.

[17] East, Johnny. IRB Approved Pilot Safety Study of an Extracellular Vesicle Isolate Product Evaluating the Treatment of Osteoarthritis in Combat-Related Injuries. Journal of Stem Cell Research. Sep. 2020; 1(2):1-10.

[18] Sengupta, Vikram, et al. Exosomes Derived from Bone Marrow Mesenchymal Stem Cells as Treatment for Severe COVID-19. Stem cells and development. Jun. 2020; 29(12): 747-754. 